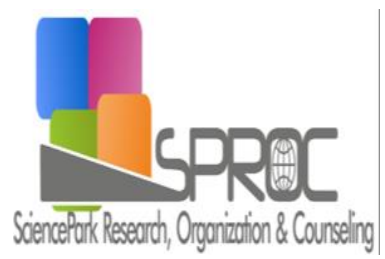

\title{
Evaluating the Weights of Criteria for Decoupling Point Positioning
}

Hande Erdogan Aktan ${ }^{\mathrm{a} *}$,

Suggested Citation:

New Trends and

Issues Proceedings on Humanities and Social Sciences.

Abstract 
1. Introduction 


\section{Leagile Supply Chain Strategy}

\section{Methodology}

\subsection{Fuzzy Dematel}

Step 1: Evaluating the criteria using fuzzy linguistic scale 
Step 2: Aggregating the assessments of decision makers and deriving average matrix: $\mathrm{Z}$

Step 3: Normalizing the direct-relation matrix:

$X=s . Z$

$s=\frac{1}{s w} i, j=1, \ldots, n$

Step 4: Attaining total-relation matrix:

$T=X(1-X)^{-}$

Step 5: Setting a threshold value and producing a causal diagram:

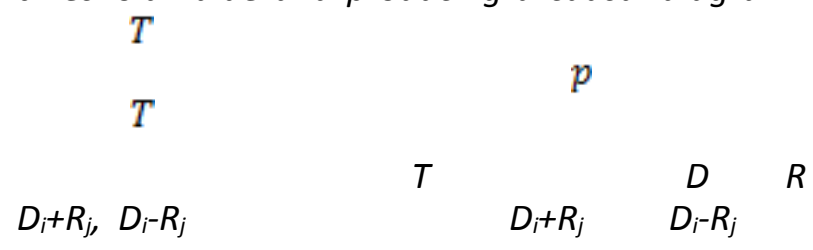

\subsection{Dematel based ANP (DANP)}

Step 1: Establishing unweighted supermatrix: $T_{C}^{\infty}$

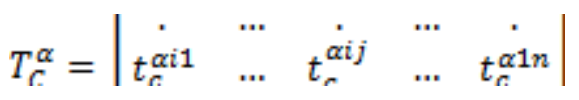

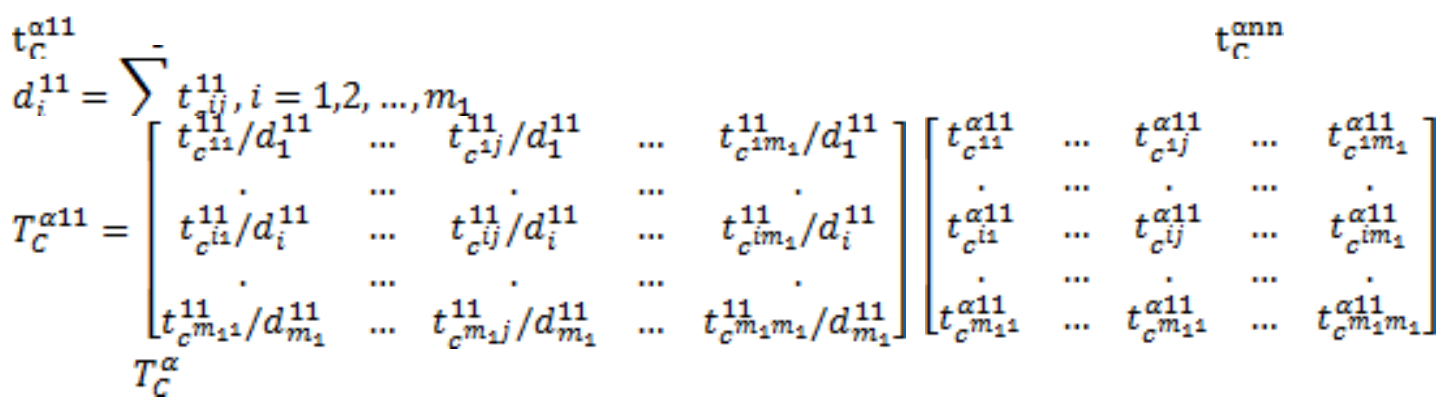




$$
W=\left(T_{C}^{\alpha}\right)^{r}=\left|\begin{array}{ccccc}
W^{1 j} & \ldots & \dot{W}^{i j} & \ldots & W^{n j}
\end{array}\right|
$$

Step 2: Obtaining weighted supermatrix:

$$
T_{D}^{\propto T_{D}}
$$

$T_{D}=\left|\begin{array}{ccccc}* & \ldots & x^{i j} & \ldots & * \\ t_{n}^{i 1} & \ldots & t_{n}^{i j} & \ldots & t_{n}^{1 n}\end{array}\right|$

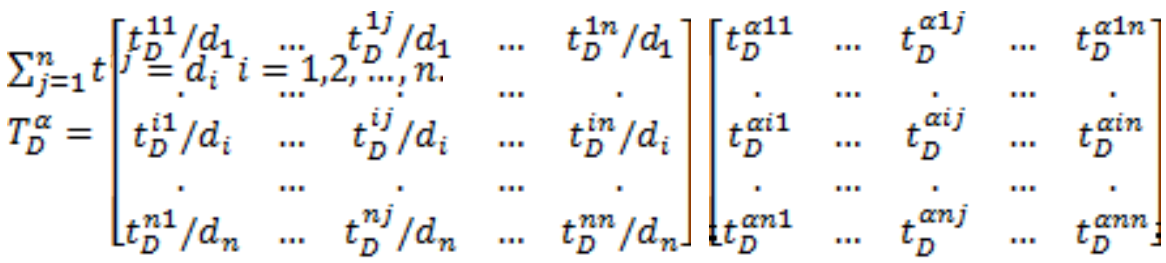

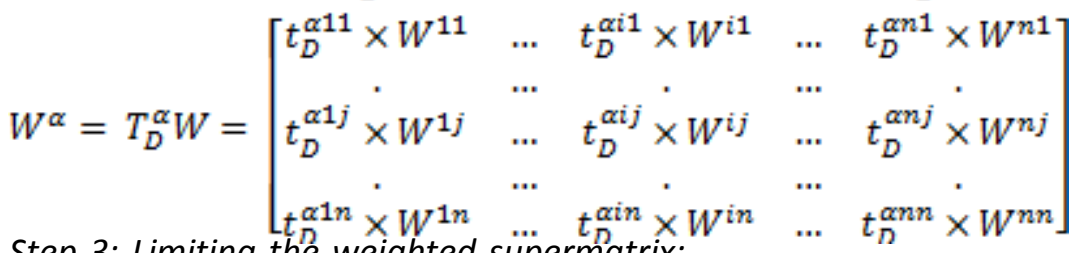

\section{Application}


$t_{i j}^{*}$

Table 1. Sum of Rows and Columns of Dimensions

\begin{tabular}{llllllll}
\cline { 2 - 6 } & $C_{1}$ & $C_{2}$ & $C_{3}$ & $D_{i}$ & $R_{j}$ & $D_{i}+R_{j}$ & $D_{i}-R_{j}$ \\
\hline$C_{1}$ & & & & & & & \\
\hline$C_{2}$ & & & & & & & \\
\hline$C_{3}$ & & & & & & & \\
\hline
\end{tabular}

$D_{i}+R_{j}$

$D_{i}-R_{j}$

$D_{i}-R_{j}$

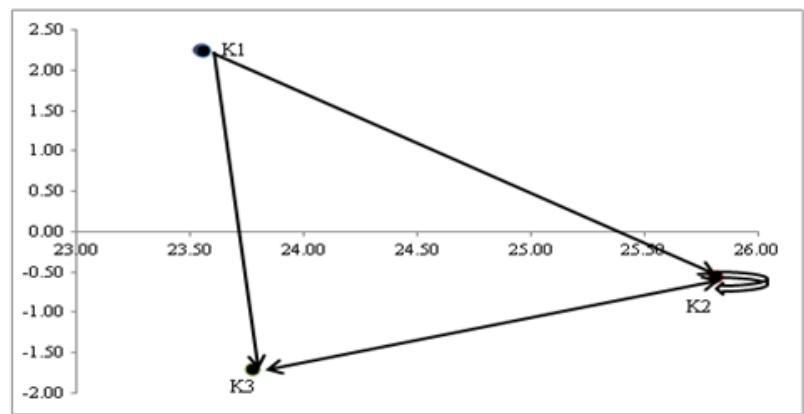

Figure 1. Causal Diagram Between Dimensions

Table 2. Matrix $T$ of All Criteria

\begin{tabular}{|c|c|c|c|c|c|c|c|c|c|c|}
\hline$[0,185$ & 0,247 & 0,374 & 0,316 & 0,340 & 0,223 & 0,342 & 0,329 & 0,312 & 0,275 & 1 \\
\hline & 0,186 & 0,386 & 0,303 & 0,318 & 0,232 & & 24 & 24 & & \\
\hline 37 & 0,368 & 0,419 & 0,444 & 451 & 0,339 & 0,477 & 0,480 & 0,474 & 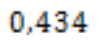 & \\
\hline & 0,260 & 17 & & & 0,263 & & & & & \\
\hline 0,297 & 0,263 & 0,4 & 40 & 0,2 & 0,262 & 0 & 44 & 32 & & \\
\hline 0.202 & 0,237 & 0,349 & 0,289 & 0.291 & 0,194 & 0,3 & & & & \\
\hline 0,276 & 0,308 & 0,481 & 2 & $0_{2} 4$ & 0,330 & $0_{s}$ & & & $0_{2}$ & \\
\hline 0,301 & 0,325 & 0,495 & 0,415 & 0,4 & 0,327 & 0,434 & 51 & & & \\
\hline 0.274 & 0,267 & 0,423 & 0,372 & 0,3 & 0,324 & 0,385 & 4 & & & \\
\hline 0,256 & 0,249 & 0,397 & 0,328 & 0,3 & 0,288 & 0,351 & $0_{2}$ & & & \\
\hline 0,263 & 0,249 & 0,391 & 0,319 & 0,330 & 0,291 & 0,345 & 0,367 & 0,368 & 0,328 & \\
\hline
\end{tabular}

$T$

Table 3. Sum of Rows and Sum of Columns of All Criteria 


\begin{tabular}{|c|c|c|c|c|c|c|c|c|c|c|c|}
\hline & $C_{11}$ & $C_{12}$ & $C_{13}$ & $C_{14}$ & $C_{15}$ & $C_{21}$ & $C_{22}$ & $C_{23}$ & $C_{31}$ & $C_{32}$ & $C_{33}$ \\
\hline \multicolumn{12}{|l|}{$\overline{D_{i}}$} \\
\hline \multicolumn{12}{|l|}{$\boldsymbol{R}_{j}$} \\
\hline \multicolumn{12}{|l|}{$D_{i}+R_{j}$} \\
\hline$D_{i}-R_{j}$ & & & & & & & & & & & \\
\hline
\end{tabular}

$R_{j}$

$D i-R j$

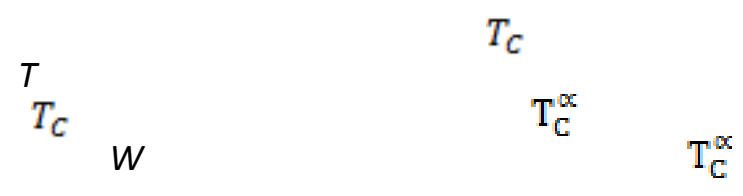

Table4. Weights of Criteria Obtained by DANP Method

\begin{tabular}{llllllllllll}
\hline $\begin{array}{l}\text { Criteria } \\
\text { (i) }\end{array}$ & $\mathrm{C}_{11}$ & $\mathrm{C}_{12}$ & $\mathrm{C}_{13}$ & $\mathrm{C}_{14}$ & $\mathrm{C}_{15}$ & $\mathrm{C}_{21}$ & $\mathrm{C}_{22}$ & $\mathrm{C}_{23}$ & $\mathrm{C}_{31}$ & $\mathrm{C}_{32}$ & $\mathrm{C}_{33}$ \\
\hline $\begin{array}{l}\text { Weight } \\
\left(W_{i}\right)\end{array}$ & & & & & & & & & & & \\
\hline
\end{tabular}


$D_{i}+R_{j}$

$D_{i}+R_{j}$

5. Conclusions

$D_{i}+R_{j}$

$D_{i}-R_{j}$

$D_{i-} R_{j}$

$D_{i-} R_{j}$

$D_{i}-R_{j}$

$D_{i}+R_{j}$

Acknowledgements 


\section{References}

Journal of Business Economics and Management, 17

Management, 7

Journal of Global Strategic

Expert Systems with Applications. 38

International Journal of Physical Distribution\&Logistics Management. 31

International Journal of Logistics Management. 13

The

Teknoloji. 8

British Food Journal, 118

International Journal of Operations \& Production Management, 29

International Journal of Advanced Manufacturing Technology, 48

Data Systems, 109

Industrial Management \&

International Journal of Fuzzy Systems, 9

and Recycling, 66

Resources, Conservation

Chain Management: An International Journal, 7

Technological Forecasting\&Social Change, 103

Sustainability, 7

International

Journal of Agile Management Systems, 2 
Journal of Production Research, 38

Journal of Applied and Emerging Sciences, 4 